\title{
Interactive Hair Rendering and Appearance Editing under Environment Lighting
}

\author{
Kun $\mathrm{Xu}^{1} \quad$ Li-Qian $\mathrm{Ma}^{1} \quad$ Bo Ren $^{1} \quad$ Rui Wang $^{2} \quad$ Shi-Min $\mathrm{Hu}^{1}$
}

${ }^{1}$ TNList, Department of Computer Science and Technology, Tsinghua University, Beijing

${ }^{2}$ Department of Computer Science, University of Massachusetts, Amherst
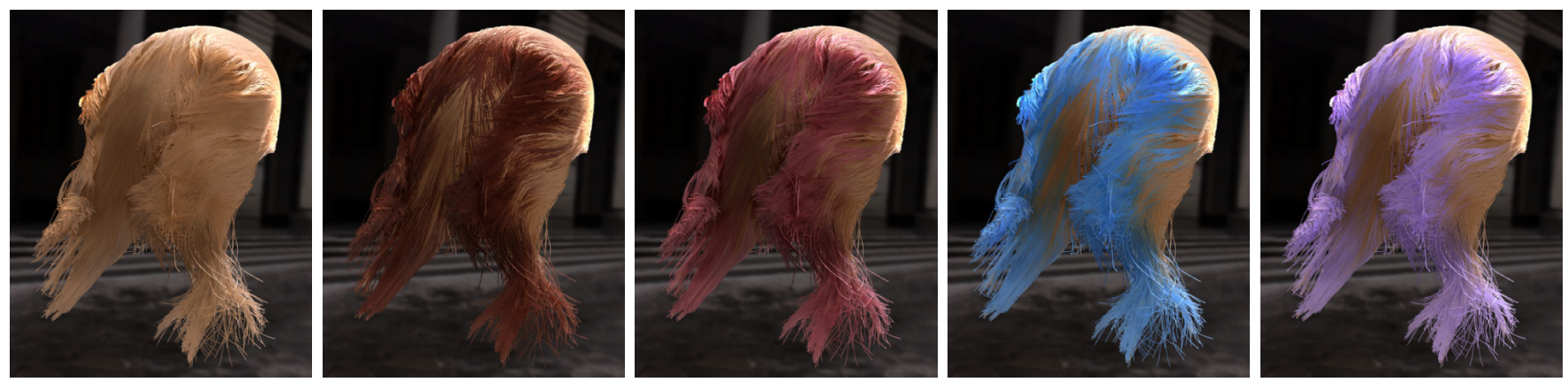

Figure 1: Our algorithm achieves interactive hair rendering and appearance editing under environment lighting, including both single and multiple scattering effects. In this example, the user directly paints onto the hair to edit the spatially-varying scattering parameters. This results in a dynamic simulation of hair coloring. From left to right, the hair is dyed with a progressively more vivid color. The environment map is represented by 40 SRBF lights, and our algorithm runs at $8.3 \mathrm{fps}$ on an NVIDIA GTX 580.

\begin{abstract}
We present an interactive algorithm for hair rendering and appearance editing under complex environment lighting represented as spherical radial basis functions (SRBFs). Our main contribution is to derive a compact $1 \mathrm{D}$ circular Gaussian representation that can accurately model the hair scattering function introduced by [Marschner et al. 2003]. The primary benefit of this representation is that it enables us to evaluate, at run-time, closed-form integrals of the scattering function with each SRBF light, resulting in efficient computation of both single and multiple scatterings. In contrast to previous work, our algorithm computes the rendering integrals entirely on the fly and does not depend on expensive precomputation. Thus we allow the user to dynamically change the hair scattering parameters, which can vary spatially. Analyses show that our 1D circular Gaussian representation is both accurate and concise. In addition, our algorithm incorporates the eccentricity of the hair. We implement our algorithm on the GPU, achieving interactive hair rendering and simultaneous appearance editing under complex environment maps for the first time.
\end{abstract}

Keywords: Hair rendering, environment lighting, appearance editing, SRBFs, circular Gaussian, single and multiple scattering, GPU.

Links: - DL 国PDF WEB

\section{Introduction}

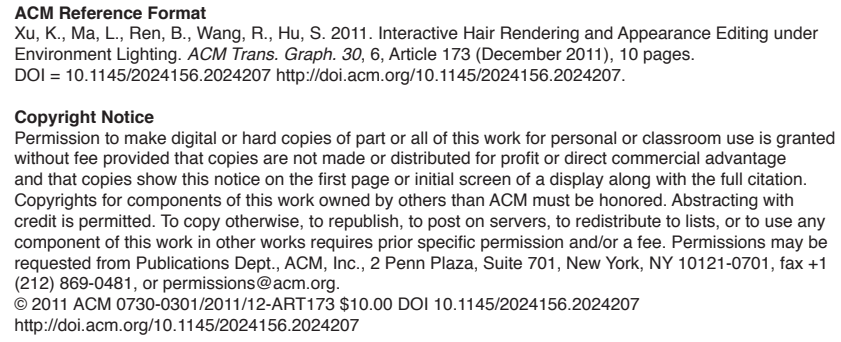

In hair rendering, it is often desirable to support the dynamic changing of hair's scattering properties. This enables artists and designers to edit hair appearance at will, and receive realistic rendering feedbacks at interactive speed. Existing methods already support such capability under simple lighting, such as a few point or directional lights [Zinke et al. 2008; Yuksel and Keyser 2008; Shinya et al. 2010; Sadeghi et al. 2010]. However, it remains a challenge to render and simultaneously edit hair appearance under complex lighting such as environment maps [Debevec and Malik 1997]. Such lighting is important to convey the rich look of the hair in natural illumination conditions.

The main difficulty with environment lighting is the large number of directional lights that must be considered. An effective solution is to approximate the environment map as a set of spherical radial basis functions (SRBFs), yielding a low-dimensional representation. This approach has been studied in the context of precomputed light transport and BRDF aprpoximation [Tsai and Shih 2006; Green et al. 2007; Wang et al. 2009]. For hair rendering, Ren et al. [2010] proposed to integrate the hair scattering function with each SRBF light to produce realistic rendering effects. Their method incorporates both single and multiple scatterings. Unfortunately, as they precompute the integrals of the scattering function with sampled SRBF lights into 4D tables, their method requires fixing hair scattering parameters at precomputation time, disabling online editing.

In this paper, our goal is to enable realistic rendering and simultaneous editing of hair appearance under complex environment lighting. Similar to previous work, we represent an environment map using SRBF lights. Our main contribution is to derive a compact 1D circular Gaussian representation that can accurately model the hair scattering function introduced by [Marschner et al. 2003]. Analyses show that our representation is both concise and accurate. Exploiting the properties of Gaussian functions, the primary benefit of this representation is that it enables the run-time evaluation of closedform integrals of the scattering function with SRBF lights. This results in efficient computation of both single and multiple scatterings, without the need of expensive precomputation. By using this approach, our algorithm evaluates the rendering integrals entirely on the fly, allowing the user to dynamically change hair scattering parameters at will. Our approach can be seen as an accurate model of the scattering function that is particularly suitable for integration 
with SRBF lights. In addition, our algorithm successfully incorporates the eccentricity of the hair, which is important for capturing the hair's rich look.

We provide a GPU implementation that achieves interactive rates for rendering and editing of spatially-varying hair appearance under environment maps. We believe the ability to dynamically adjust the hair appearance under natural lighting provides artists a convenient way for designing hairs. Our method is the first to achieve such capability, including the editing of spatially-varying hair parameters and hair eccentricity effects. Figure 1 shows an example of our results captured at run-time.

\section{Related works}

Hair Rendering has attracted significant research attention over the years. A comprehensive survey can be found in [Ward et al. 2007]. Some of the early work focused on studying how a single hair fiber reflects the light, which can be described by a hair scattering function. Kajiya and Kay [1989] proposed the first hair scattering function, which has been a simple and popular model in many applications. Marschner et al. [2003] proposed a more accurate model, inspired by the measurements of real human hair fibers. Under distant lighting, they model each hair fiber as a dielectric cylinder and derive a scattering function that consists of three light paths: $\mathrm{R}$, TT, and TRT (see Fig 2(b)). Zinke and Weber [2007] proposed a more general model that allows for near-field lighting. A number of recent papers have also studied how to estimate hair scattering parameters and geometry from a single photograph [Zinke et al. 2009; Bonneel et al. 2009] or from real hairs using a sophisticated capturing device [Paris et al. 2008; Jakob et al. 2009].

For interactive hair rendering, many existing methods have successfully addressed the issue of self-shadowing in a large number of hair fibers. Some representative techniques include deep shadow maps [Lokovic and Veach 2000], density clustering [Mertens et al. 2004], opacity shadow maps [Kim and Neumann 2001; Sintorn and Assarsson 2008], deep opacity maps [Yuksel and Keyser 2008], and occupancy maps [Sintorn and Assarsson 2009].

As light can bounce many times inside the hair, realistic hair rendering requires the accurate simulation of multiple scattering effects, which are particularly important for light colored hair. Standard path tracing is too expensive for computing multiple scattering, thus researchers have exploited photon mapping [Moon and Marschner 2006; Zinke and Weber 2006] and spherical harmonics approximation [Moon et al. 2008] to greatly improve offline rendering speed. In [2008], Zinke et al. introduced a dual scattering approximation that achieves real-time rendering of multiple scattering. Using this model, Ren et al. [2010] proposed a technique to support interactive hair rendering under environment lighting represented as SRBF lights. This produces rich hair appearance under natural illumination. However, their technique requires fixing scattering parameters offline and ignores the eccentricity of the hair. An alternative way to compute multiple scattering effects is by using a plane-parallel model, as proposed by [Shinya et al. 2010]. While this method is fast under simple lighting, it is still very expensive for rendering under area or environment lights.

Recently, Sadeghi et al. [2010] proposed an artist-friendly control (AFC) model for intuitive editing of hair parameters. Their method assumes simple lighting conditions. As their model is fundamentally based on Marschner's physically-based scattering function, and the dual scattering approximation, it can be combined with our method in order to provide an intuitive hair editing framework. d'Eon et al. [2011] proposed an energy-conserving hair scattering model by considering higher order light paths and using nonGaussian longitudinal lobes.
SRBF approximates a function defined on the sphere using spherical Gaussians. Compared to other bases such as spherical harmonics and wavelets, SRBF provide excellent local support as well as closed-form solutions for computing product integrals. Therefore it proves to be an excellent choice for representing environment maps and BRDFs [Tsai and Shih 2006; Green et al. 2007; Wang et al. 2009]. Our work uses the circular Gaussian, which is a $1 \mathrm{D}$ version of SRBF and thus shares the same benefits. Additionally, it allows us to formulate the integration involving hair scattering functions as $1 \mathrm{D}$ integrals, enabling fast and accurate computation.

Material Editing under Environment Maps, such as BRDF editing [Ben-Artzi et al. 2006; Sun et al. 2007; Wang et al. 2009], refractive material editing [Sun et al. 2008], translucent material editing [Xu et al. 2007; Wang et al. 2008] and dynamic participating media [Navarro et al. 2009], has drawn a lot of attention in recent years. As an addition to them, ours is the first to enable hair material editing under environment maps.

\section{Background}

Terminology. For notations and symbols we follow [Marschner et al. 2003] and [Ren et al. 2010]. Fig 2(a) shows the local geometry of a hair fiber. We use $\omega_{i}=\left(\theta_{i}, \phi_{i}\right)$ to denote the lighting direction, and $\omega_{o}=\left(\theta_{o}, \phi_{o}\right)$ to denote the viewing direction. All directions and angles are expressed in the local frame of the hair fiber. Table 1 lists the set of symbols that we use throughout the paper.

Lighting Approximation. As in [Ren et al. 2010] and [Tsai and Shih 2006], an environment map $L$ is approximated as the sum of a set of SRBFs. Using $j$ as the index of an SRBF light:

$$
L\left(\omega_{i}\right) \approx \sum_{j} L_{j} G\left(\omega_{i} ; \omega_{j}, \lambda_{j}\right)
$$

where $L_{j}$ is the coefficient, $G\left(\omega_{i} ; \omega_{j}, \lambda_{j}\right)=\exp \left(2\left(\omega_{i} \cdot \omega_{j}-1\right) / \lambda_{j}^{2}\right)$ is an SRBF centered at $\omega_{j}$ with bandwidth $\lambda_{j}$. In the following we simply write it as $G_{j}\left(\omega_{i}\right)$. Note that the bandwidth $\lambda_{j}$ is defined equivalently but expressed differently from [Ren et al. 2010]. The purpose is to make it consistent with 1D circular Gaussians, which we will use later to approximate the hair's scattering function.

Single Scattering. Following [Ren et al. 2010], the single scattering radiance viewed from direction $\omega_{o}$ under a set of SRBF lights can be approximated as:

$$
L\left(\omega_{o}\right) \approx D \sum_{j} L_{j} \widetilde{T}\left(\omega_{j}, \lambda_{j}\right) \int_{\Omega} G_{j}\left(\omega_{i}\right) S\left(\omega_{i}, \omega_{o}\right) \cos \theta_{i} d \omega_{i}
$$

where $D$ is the hair fiber's diameter, $S\left(\omega_{i}, \omega_{o}\right)$ is the bidirectional scattering function, and $\widetilde{T}\left(\omega_{j}, \lambda_{j}\right)$ is the effective transmittance, estimated using a summed area table (SAT) built from a convolution optimal depth map. Essentially $\widetilde{T}$ measures the average attenuation of an SRBF light $j$ due to transmission of the light through the hair.

Multiple Scattering. Using a dual scattering model [Zinke et al. 2008], Ren et al. [2010] approximate the multiple scattering radiance (denoted as $L^{D}$ ) under environment lighting as:

$$
L^{D}\left(\omega_{o}\right) \approx D \sum_{j} L_{j} T_{f}\left(\omega_{j}\right) \int_{\Omega} \psi_{f}(\cdot) S^{D}\left(\omega_{i}, \omega_{o}\right) \cos \theta_{i} d \omega_{i}
$$

where $T_{f}\left(\omega_{j}\right)$ is the forward scattering transmittance along the shadow path of light $j$, and $S^{D}\left(\omega_{i}, \omega_{o}\right)$ is the Bidirectional Curves Scattering Distribution Function (BCSDF). The main idea of dual scattering is to estimate $S^{D}$ as the sum of a global scattering component and a local scattering component, thus:

$$
S^{D}\left(\omega_{i}, \omega_{o}\right)=S\left(\omega_{i}, \omega_{o}\right)+d_{b} S_{\text {back }}\left(\omega_{i}, \omega_{o}\right)
$$




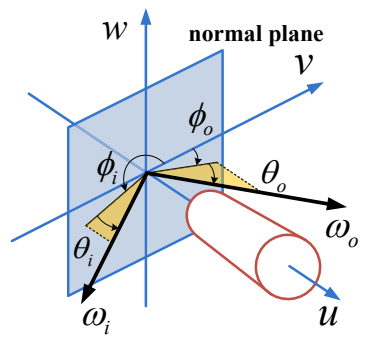

(a)

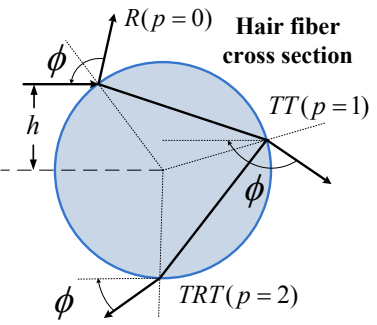

(b)
Figure 2: Illustration of (a) notations, and (b) scattering paths.

where $S$ is the bidirectional scattering function and $S_{\text {back }}$ is the backward scattering function accounting for local scattering. $d_{b}$ is a constant set between $0.6-0.8$. Back to $\operatorname{Eq} 3, \psi_{f}(\cdot)$ is the spread function that approximates the final angular distribution of the front scattered radiance, and is computed as:

$$
\psi_{f}(\cdot)=\tilde{s}_{f}\left(\phi_{j}, \phi_{i}\right) g^{u}\left(\theta_{i} ; \theta_{j}, \sigma_{f}\left(\omega_{j}, \lambda_{j}\right)\right) / \cos \theta_{j}
$$

where $\theta_{j}, \phi_{j}$ are the inclination and azimuth angles of $\omega_{j} ; \tilde{s}_{f}\left(\phi_{j}, \phi_{i}\right)$ is defined as $1 / \pi$ for forwarding scattering and 0 for backward scattering; and $g^{u}\left(\theta_{i} ; \theta_{j}, \sigma_{f}\right)$ is a normalized 1D Gaussian function of $\theta_{i}$ centered at $\theta_{j}$ with bandwidth $\sigma_{f}$. Here $\sigma_{f}=\sqrt{ }\left[\lambda_{j}^{2}+\bar{\sigma}_{f}^{2}\left(\omega_{j}\right)\right]$, where $\bar{\sigma}_{f}^{2}\left(\omega_{j}\right)$ is the average forward scattering spread.

Summary of [Ren et al. 2010]. As the integrals involved in Eq. 2 and 3 are independent of hair geometry, they can be precomputed into 4D tables by sampling all possible SRBFs offline. At run-time, the effective transmittance $\widetilde{T}$ (in single scattering) is approximated by convolution optical depth maps; the forward scattering transmittance $T_{f}$ and spread $\sigma_{f}$ (in multiple scattering) are evaluated by interpolation from sparse samples; and then the two integrals in Eq. 2 and 3 can be estimated by querying the precomputed 4D tables. This allows for interactive hair rendering with $30 \sim 60 \mathrm{SRBF}$ lights. Unfortunately, as the hair scattering coefficients are baked into the precomputed tables, which take hours to compute, dynamic editing of these coefficients is not possible. In addition, it's unclear how to incorporate the hair eccentricity into their approach.

\section{Algorithm Details}

Overview. This section describes our algorithms. We first review the hair scattering model by Marschner et al. [2003], then derive a compact 1D circular Gaussian representation to approximate the model. We discuss the algorithms for rendering single scattering and multiple scattering separately in Section 4.2 and 4.3.

Key idea. On a high level, our key idea is that an SRBF (i.e. the lighting basis) can be separated into the product of two 1D circular Gaussians in the two spherical coordinates $\theta$ and $\phi$ (Eq. 11). This matches the formulation of the hair scattering model, which is also written as the product of a longitudinal $(\theta)$ function and an azimuthal $(\phi)$ function (Eq. 6). The separation into 1D functions allows us to quickly compute the rendering integral on the fly, by using the analytic properties of Gaussians and the smoothness of several run-time evaluated terms. Specifically, we exploit the property that the product of two Gaussians is still a Gaussian, and we introduce a simple 1D quadrature method to evaluate the integral of a Gaussian with a smooth function (see Section 4.2.2). Finally, the $1 \mathrm{D}$ representation also allows us to precompute certain integrals into small 2D tables, which are used for run-time lookups.

\subsection{Hair Scattering Model}

Derived in [Marschner et al. 2003], the hair scattering function $S\left(\omega_{i}, \omega_{o}\right)$ is the sum of three modes: $S_{R}$ (reflection), $S_{T T}$ (transmission-transmission), and $S_{T R T}$ (transmission-reflectiontransmission). Thus $S\left(\omega_{i}, \omega_{o}\right)=\sum_{t} S_{t}\left(\omega_{i}, \omega_{o}\right)$, where the mode in$\operatorname{dex} t \in\{R, T T, T R T\}$. Each term $S_{t}$ is further represented as the product of a longitudinal function $M_{t}$ and an azimuthal function $N_{t}$ :

$$
S_{t}\left(\omega_{i}, \omega_{o}\right)=M_{t}\left(\theta_{h}\right) N_{t}\left(\eta, \theta_{d}, \phi\right) / \cos ^{2} \theta_{d}
$$

where $\theta_{h}, \theta_{d}$ and $\phi$ are the half and difference angles, and $\eta$ is the index of refraction. The longitudinal function $M_{t}$ is defined by:

$$
M_{t}\left(\theta_{h}\right)=g^{u}\left(\theta_{h} ; \alpha_{t}, \beta_{t}\right)=2 g^{u}\left(\theta_{i} ; 2 \alpha_{t}-\theta_{o}, 2 \beta_{t}\right)
$$

which is a normalized 1D Gaussian of $\theta_{i}$ centered at $2 \alpha_{t}-\theta_{o}$ with bandwidth $2 \beta_{t}$. Here $\alpha_{t}$ and $\beta_{t}$ are the longitudinal shift and bandwidth of each mode, caused by the tilting of the hair scales.

The azimuthal function $N_{t}$ is derived by examining the scattering paths through a circular cross profile, shown in Figure 2(b). First, an analysis of reflections and refractions of a ray through the circle reveals that the azimuthal difference angle $\phi=\phi_{o}-\phi_{i}$ can be expressed as a function of the incident direction offset $h$ :

$$
\phi(p, h)=2 p \sin ^{-1}\left(h / \eta^{\prime}\left(\eta, \theta_{d}\right)\right)-2 \sin ^{-1}(h)+p \pi
$$

where $\eta^{\prime}$ is the effective index of refraction (see Table 1), $p$ is the number of internal reflections. It is 0 for the R mode, 1 for the TT mode and 2 for the TRT mode. This allows the azimuthal scattering function $N_{t}$ to be defined as:

$$
N_{t}\left(\eta, \theta_{d}, \phi\right)=\sum_{h} \frac{1}{2}\left\|\frac{d \phi}{d h}\right\|^{-1} A_{t}\left(\theta_{d}, h\right)
$$

which sums over all $h$ 's satisfying $\phi(p, h)=\phi$. Here $A_{t}$ is given as:

$$
\begin{aligned}
A_{R}\left(\theta_{d}, h\right) & =F\left(\eta, \theta_{d}, h\right) \\
A_{T T}\left(\theta_{d}, h\right) & =\left(1-F\left(\eta, \theta_{d}, h\right)\right)^{2} T\left(\sigma_{a}^{\prime}\left(\theta_{d}\right), h\right) \\
A_{T R T}\left(\theta_{d}, h\right) & =\left(1-F\left(\eta, \theta_{d}, h\right)\right)^{2} F\left(\eta, \theta_{d}, h\right) T^{2}\left(\sigma_{a}^{\prime}\left(\theta_{d}\right), h\right)
\end{aligned}
$$

where $F$ is the Fresnel reflection term, $\sigma_{a}^{\prime}$ is the effective absorption coefficient (see Table 1), $T$ is an attenuation factor due to absorption on the internal paths, and is computed by $T\left(\sigma_{a}^{\prime}\left(\theta_{d}\right), h\right)=$ $\exp \left(-2 \sigma_{a}^{\prime} \sqrt{1-\frac{h^{2}}{\eta^{\prime 2}}}\right)$.

\subsection{Computing Single Scattering}

Substitute the scattering mode function defined in Eq 6 to the single scattering integral in Eq 2, and define the result as $\mathscr{M}_{t}$, we have:

$$
\begin{aligned}
& \mathscr{M}_{t}\left(\omega_{j}, \lambda_{j}\right)=\int_{\Omega} G_{j}\left(\omega_{i}\right) S_{t}\left(\omega_{i}, \omega_{o}\right) \cos \theta_{i} d \omega_{i} \\
= & \int_{\Omega} G_{j}\left(\omega_{i}\right) M_{t}\left(\theta_{h}\right) N_{t}\left(\theta_{d}, \phi\right) \cos \theta_{i} / \cos ^{2} \theta_{d} d \omega_{i}
\end{aligned}
$$

By expressing the SRBF $G_{j}$ in spherical coordinates $\theta$ and $\phi$, we can decompose it into the product of two 1D circular Gaussians (refer to Appendix), and expand the integral over $\Omega$ to double integrals over $\theta_{i}$ and $\phi_{i}$ :

$$
\begin{aligned}
& \mathscr{M}_{t}\left(\omega_{j}, \lambda_{j}\right)=\iint\left(g_{j}^{c}\left(\theta_{i}\right) g_{j}^{c}\left(\phi_{i}\right)\right) M_{t}\left(\theta_{h}\right) N_{t}\left(\theta_{d}, \phi\right) \frac{\cos ^{2} \theta_{i}}{\cos ^{2} \theta_{d}} d \phi_{i} d \theta_{i} \\
= & \int g_{j}^{c}\left(\theta_{i}\right) M_{t}\left(\theta_{h}\right) \frac{\cos ^{2} \theta_{i}}{\cos ^{2} \theta_{d}}\left(\int g_{j}^{c}\left(\phi_{i}\right) N_{t}\left(\theta_{d}, \phi\right) d \phi_{i}\right) d \theta_{i} \\
\approx & \int g_{j}\left(\theta_{i}\right) M_{t}\left(\theta_{h}\right) \frac{\cos ^{2} \theta_{i}}{\cos ^{2} \theta_{d}} \mathscr{N}_{t}\left(\lambda_{j}^{\prime}, \phi_{o}-\phi_{j}, \theta_{d}\right) d \theta_{i}
\end{aligned}
$$




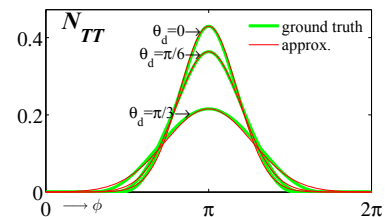

(a) Approximation of $N_{T T}$

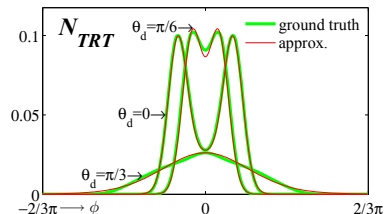

(b) Approximation of $N_{T R T}$

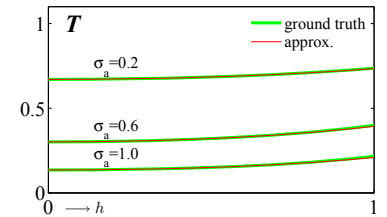

(c) Approximation of $T\left(\sigma_{a}^{\prime}\left(\theta_{d}\right), h\right)$

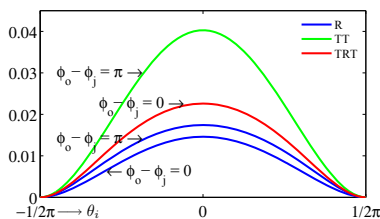

(d) Plot of $\mathscr{N}_{t} \cos ^{2} \theta_{i} / \cos ^{2} \theta_{d}$

Figure 3: (a): approximating $N_{T T}$ using a single circular Gaussian (Eq 16); (b): approximating $N_{T R T}$ using the sum of three circular Gaussians (Eq 20); (c): approximating $T\left(\sigma_{a}^{\prime}\left(\theta_{d}\right), h\right)$ using 4-th order Taylor expansion (Eq 18); (d): Plot of $\mathscr{N}_{t} \cos ^{2} \theta_{i} / \cos ^{2} \theta_{d}, t \in(R, T T, T R T)$.

\begin{tabular}{ll}
\hline$\eta, \sigma_{a}$ & index of refraction, and absorption coefficient \\
$\alpha_{R}, \alpha_{T T}, \alpha_{T R T}$ & longitudinal shift for R,TT,TRT lobes \\
$\beta_{R}, \beta_{T T}, \beta_{T R T}$ & longitudinal width for R,TT,TRT lobes \\
$a, w_{c}$ & eccentricity, and azimuthal width of TRT lobes \\
$\theta, \phi$ & longitudinal and azimuthal angles \\
$\omega_{i}, \omega_{o}$ & incoming and viewing directions \\
$\omega_{j}, \lambda_{j}$ & center and bandwidth of an SRBF light $j$ \\
$\theta_{d}=\left(\theta_{o}-\theta_{i}\right) / 2 ; \theta_{h}=\left(\theta_{o}+\theta_{i}\right) / 2 ; \phi=\phi_{o}-\phi_{i} ; \phi_{h}=\left(\phi_{o}+\phi_{i}\right) / 2$ \\
\hline$h \in(-1,1)$ & incident direction offset \\
$\eta^{\prime}=\sqrt{ }\left[\eta^{2}-\sin ^{2} \theta_{d}\right] / \cos \theta_{d}$ & effective index of refraction \\
$\lambda_{j}^{\prime}=\lambda j / \sqrt{ }\left[\cos \theta_{i} \cos \theta_{j}\right]$ & effective Gaussian bandwidth \\
$\sigma_{a}^{\prime}=\sigma_{a} / \sqrt{ }\left[1-\sin ^{2} \theta_{d} / \eta^{2}\right]$ & effective absorption coefficient \\
$g(x ; \mu, \lambda)=\exp \left[-(x-\mu)^{2} / \lambda^{2}\right]$ & 1D Gaussian \\
$g^{u}(x ; \mu, \lambda)=g(x ; \mu, \lambda) /(\sqrt{\pi} \lambda)$ & normalized 1D Gaussian \\
$g^{c}(x ; \mu, \lambda)=\exp \left[\frac{2(\cos (x-\mu)-1)}{\lambda^{2}}\right]$ & 1D circular Gaussian \\
$G\left(\omega ; \omega_{j}, \lambda\right)=\exp \left[\frac{2\left(\omega \cdot \omega_{j}-1\right)}{\lambda^{2}}\right]$ & SRBF \\
\hline
\end{tabular}

Table 1: List of symbols and notations used in this paper.

where $g_{j}^{c}\left(\theta_{i}\right)=g^{c}\left(\theta_{i} ; \theta_{j}, \lambda_{j}\right)=\exp \left[2\left(\cos \left(\theta_{i}-\theta_{j}\right)-1\right) / \lambda_{j}^{2}\right]$ and $g_{j}^{c}\left(\phi_{i}\right)=g^{c}\left(\phi_{i} ; \phi_{j}, \lambda_{j}^{\prime}\right)=\exp \left[2\left(\cos \left(\phi_{i}-\phi_{j}\right)-1\right) / \lambda_{j}^{\prime 2}\right]$ are two 1D circular Gaussians whose product equals $G_{j}\left(\omega_{i}\right)$. Here the effective azimuthal Gaussian bandwidth $\lambda_{j}^{\prime}=\lambda_{j} / \sqrt{\cos \theta_{i} \cos \theta_{j}}$. Note that the last step of Eq 11 uses $\mathscr{N}_{t}$ to denote the inner integral over $d \phi_{i}$; also, it uses the fact that the 1D circular Gaussian $g_{j}^{c}\left(\theta_{i}\right)$ is well approximated by a 1D Gaussian $g_{j}\left(\theta_{i}\right)=g\left(\theta_{i} ; \theta_{j}, \lambda_{j}\right.$ ) (proof is shown in Section 1 of the supplemental document). By changing the integral variable from $\phi_{i}$ to $\phi_{i}^{\prime}=\phi_{i}-\phi_{j}, \mathscr{N}_{t}$ can be rewritten as:

$$
\mathscr{N}_{t}\left(\lambda_{j}^{\prime}, \phi_{o}-\phi_{j}, \theta_{d}\right)=\int g^{c}\left(\phi_{i}^{\prime} ; 0, \lambda_{j}^{\prime}\right) N_{t}\left(\theta_{d}, \phi_{o}-\phi_{j}-\phi_{i}^{\prime}\right) d \phi_{i}^{\prime}
$$

Essentially Eq 11 has turned the original rendering integral to two 1D integrals: the inner integral $\mathscr{N}_{t}$ and the outer integral $\mathscr{M}_{t}$. In the following we will explain how to evaluate these two integrals for the three scattering modes $t \in R, T T, T R T$ respectively.

\subsubsection{Approximating the $\mathrm{R}$ Mode}

In the R Mode ( $p=0)$, the relationship of $\phi$ and $h$ (Eq 8) is $\phi=$ $-2 \sin ^{-1} h$, and hence $h=-\sin (\phi / 2)$. Given this, the azimuthal scattering function (Eq 9 ) can be written as:

$$
N_{R}\left(\eta, \theta_{d}, \phi\right)=\frac{1}{4}|\cos (\phi / 2)| F\left(\eta, \theta_{d},-\sin (\phi / 2)\right)
$$

The Fresnel term is defined using Schlick's approximation [1994]: $F\left(\eta, \theta_{d}, h\right) \approx F_{0}+\left(1-F_{0}\right)\left(1-\cos \theta_{d} \sqrt{1-h^{2}}\right)^{5}$, where $F_{0}=(1-$ $\eta)^{2} /(1+\eta)^{2}$ is the Fresnel value at 0 incident angle. Now $N_{R}$ can be rewritten as a polynomial of $\cos (\phi / 2)$ up to degree 6 :

$$
N_{R}\left(\eta, \theta_{d}, \phi\right) \approx \sum_{0 \leq k \leq 6} C_{k}\left(\theta_{d}, \eta\right)\left|\cos ^{k}(\phi / 2)\right|
$$

where each coefficient $C_{k}\left(\theta_{d}, \eta\right)$ is simply a polynomial of $\cos \theta_{d}$. Substituting $N_{R}$ (Eq 13) to the inner integral (Eq 12) yields:

$$
\begin{aligned}
\mathscr{N}_{R} & \approx \sum_{k} C_{k}\left(\theta_{d}, \eta\right) \int_{-\pi}^{\pi}\left|\cos ^{k}\left(\frac{\phi_{o}-\phi_{j}-\phi_{i}^{\prime}}{2}\right)\right| g^{c}\left(\phi_{i}^{\prime} ; 0, \lambda_{j}^{\prime}\right) d \phi_{i}^{\prime} \\
& =\sum_{k} C_{k}\left(\theta_{d}, \eta\right) \mathscr{C}_{k}\left(\lambda_{j}^{\prime}, \phi_{o}-\phi_{j}\right)
\end{aligned}
$$

where $\mathscr{C}_{k}$ is the product integral of the $k$-th power cosine function with a circular Gaussian $g^{c}$. Since it only depends on $\lambda_{j}^{\prime}$ and $\left(\phi_{o}-\right.$ $\phi_{j}$ ), and not on any scattering coefficient, it can be precomputed as a 2D table and accessed online through table lookups. If the user changes scattering parameters such as $\eta$, the coefficients $C_{k}\left(\theta_{d}, \eta\right)$ will be updated, but this is very fast to evaluate on the fly.

Now, let's look back at Eq 11 to see how to calculate the outer integral $\mathscr{M}_{R}$. First, note that in that equation, the longitudinal function $M_{t}$ is a $1 \mathrm{D}$ Gaussian given by Eq 7, thus its product with another 1D Gaussian $g_{j}\left(\theta_{i}\right)$ is still a Gaussian (proof is given in Section 2 of the supplemental document). Next, the remaining factor in the integral, $\cos ^{2} \theta_{i} / \cos ^{2} \theta_{d} \mathscr{N}_{R}\left(\lambda_{j}^{\prime}, \phi_{o}-\phi_{j}, \theta_{d}\right)$, is smooth as a function of $\theta_{i}$. This can be verified from our experiments in Fig 3(d). Thus its product integral with a 1D Gaussian can be accurately estimated using a numerical quadrature as detailed in the section below. In general, the numerical integration only requires a few samplings of $\mathscr{N}_{R}$ at run-time, which can be quickly evaluated using Eq 14.

\subsubsection{Product Integral with 1D Gaussian}

Given a 1D Gaussian $g(x ; \mu, \lambda)$ and an arbitrary function $f(x)$, their product integral over the range $\left[r_{0}, r_{1}\right]$ is defined as:

$$
\int_{r_{0}}^{r_{1}} g(x ; \mu, \lambda) \cdot f(x) d x
$$

In general, this integral has no analytic solution. However, if $f(x)$ is smooth, it can be numerically estimated using a piecewise linear approximation of $f(x)$. To do so, consider taking $m+1$ samples $x_{s}$, $s \in[0, m]$ and approximate $f(x)$ on each segment $\left[x_{s}, x_{s+1}\right]$ as $f(x) \approx$ $b_{s} x+c_{s}$, where $b_{s}$ and $c_{s}$ are the linear coefficients calculated from $f\left(x_{s}\right)$ and $f\left(x_{s+1}\right)$. We can now rewrite the product integral as:

$$
\sum_{0 \leq s<m} \int_{x_{s}}^{x_{s+1}} g(x ; \mu, \lambda) \cdot\left(b_{s} x+c_{s}\right) d x
$$

which can be easily computed because the product integral of a 1D Gaussian with any polynomial function has an analytic solution (this property is proved in Section 3 of the supplemental document).

Clearly the number of samples $m+1$ affects the accuracy of this approximation. In our case, since we always integrate over $[-\pi / 2, \pi / 2]$, we simply compute $m+1$ samples by picking the first $m-1$ samples uniformly in the range $(\mu-(m-2) \lambda / 2)$ to $(\mu+(m-2) \lambda / 2)$, plus the two endpoints $-\pi / 2$ and $\pi / 2$. We have found that choosing $m=4$ is usually sufficient for the hair scattering function. Fig 5 provides a comparison when using different number of samples. If desired, higher accuracy can be achieved by 
increasing the number of samples, or using a higher order polynomial approximation.

\subsubsection{Approximating the TT Mode}

In the TT mode $(p=1)$, the relationship of $\phi$ and $h$ in Eq 8 is: $\phi=2 \sin ^{-1}\left(h / \eta^{\prime}\right)-2 \sin ^{-1} h+\pi$. From Eq 10 and Eq 9, we have:

$$
N_{T T}\left(\eta, \theta_{d}, \sigma_{a}, \phi\right)=\frac{1}{2}\left|\frac{d \phi}{d h}\right|^{-1}\left(1-F\left(\theta_{d}, h\right)\right)^{2} T\left(\sigma_{a}, \theta_{d}, h\right)
$$

Our experiments in Fig 3(a) show that in practice the function $N_{T T}$ can be very well approximated using a single circular Gaussian centered at $\phi=\pi$, with coefficient $b_{t t}$ and bandwidth $\lambda_{t t}$. Thus:

$$
N_{T T}\left(\eta, \theta_{d}, \sigma_{a}, \phi\right) \approx b_{t t}\left(\eta, \theta_{d}, \sigma_{a}\right) g^{c}\left(\phi ; \pi, \lambda_{t t}\left(\eta, \theta_{d}, \sigma_{a}\right)\right)
$$

For $b_{t t}$, we set it directly as the value of $N_{T T}$ at $\phi=\pi$. To set the bandwidth $\lambda_{t t}$, note that $\int_{-\pi}^{\pi} g^{c}\left(\phi, \pi, \lambda_{t t}\right) d \phi \approx \sqrt{\pi} \lambda_{t t}$ (see Section 4 of the supplemental document), thus we estimate $\lambda_{t t}$ by preserving the energy of $N_{T T}$ :

$$
\lambda_{t t}=\frac{1}{\sqrt{\pi} b_{t t}} \int_{-\pi}^{\pi} N_{T T}\left(\eta, \theta_{d}, \sigma_{a}, \phi\right) d \phi
$$

To estimate $\int_{-\pi}^{\pi} N_{T T}$, observe that in Eq 15 the attenuation function $T$ depends on $h$. Because this function is typically smooth, we can use a 4-th order Taylor expansion to factor out its dependency on $h$ :

$$
T\left(\sigma_{a}, \theta_{d}, h\right) \approx \sum_{k \in\{0,2,4\}} a_{k}\left(\theta_{d}, \sigma_{a}\right) h^{k}
$$

where each $a_{k}\left(\theta_{d}, \sigma_{a}\right)$ is a Taylor expansion coefficient and has an analytic expression. Fig 3(c) shows the error caused by using the Taylor expansion. From the figure, we can see that the approximation error is generally very small. Combining Eq 18 and 15, we can evaluate $\int_{-\pi}^{\pi} N_{T T}$ as (note that in the following derivation, we have changed the integral variable from $d \phi$ to $d h$ ):

$$
\begin{aligned}
& \frac{1}{2} \int_{-1}^{1}\left(1-F\left(\eta, \theta_{d}, h\right)\right)^{2}\left(\sum_{k \in\{0,2,4\}} a_{k}\left(\theta_{d}, \sigma_{a}\right) h^{k}\right) d h \\
= & \frac{1}{2} \sum_{k \in\{0,2,4\}} a_{k}\left(\theta_{d}, \sigma_{a}\right) \int_{-1}^{1}\left(1-F\left(\eta, \theta_{d}, h\right)\right)^{2} h^{k} d h
\end{aligned}
$$

We denote the integral $\int_{-1}^{1}\left(1-F\left(\eta, \theta_{d}, h\right)\right)^{2} h^{k} d h$ in the above equation as $\mathscr{H}_{k}^{T T}\left(\eta, \theta_{d}\right)$. Note that each $\mathscr{H}_{k}^{T T}(k=0,2,4)$ can be precomputed and stored as a 2D table of $\eta$ and $\theta_{d}$.

While we could also estimate $\lambda_{t t}$ by sampling $N_{T T}$ at a few places, we found our energy-preserving estimation (Eq 17) is more accurate and in fact cheaper to compute on the fly. Now that function $N_{T T}$ is approximated by a single circular Gaussian (Eq 16), we can substitute $N_{T T}$ to Eq 12 to evaluate $\mathscr{N}_{T T}$. Since the product of two circular Gaussians is still a circular Gaussian (see Section 5 of the supplemental document), the integral can be easily computed using an analytic solution. Thus we have completed the evaluation of $\mathscr{N}_{T T}$.

The outer integral $\mathscr{M}_{T T}$ (Eq 11) is a product integral between a Gaussian $g_{j}\left(\theta_{i}\right)$ and function $\mathscr{N}_{T T} \cos ^{2} \theta_{i} / \cos ^{2} \theta_{d}$, which is typically smooth. This is in the same form with $\mathscr{M}_{R}$ discussed in Section 4.2.1, therefore it can be evaluated in the same way as $\mathscr{M}_{R}$.

\subsubsection{Approximating the TRT Mode}

In the TRT mode, the azimuthal function $N_{T R T}$ is more complex. As described by [Marschner et al. 2003], it is symmetric around $\phi=0$ and contains two peaks when $\eta^{\prime}<2$. The two peaks will gradually merge into one single peak centered at $\phi=0$ when $\eta^{\prime}$ increases beyond 2. When $\eta^{\prime}<2$, directly using Eq 9 will result in infinite values at the two peaks. Since the hair surface is typically rough, Marschner et al. proposed to replace the two peaks with finite-valued Gaussian functions centered at the peak locations. Fig 3(b) plots $N_{T R T}$ under different $\theta_{d}$ (hence $\eta^{\prime}$ ) values.

Our method employs a similar way to remove the infinite values. As in [Marschner et al. 2003], when $\eta^{\prime}<2$, we use two Gaussians centered at the peak locations; however, we use an additional Gaussian centered at $\phi=0$ to approximate the residue, resulting in a 3-Gaussian approximation. Detailed are discussed below.

When $\eta^{\prime}<2$. $N_{T R T}$ is approximated as the sum of 3 Gaussians:

$$
N_{T R T} \approx b_{1}\left(g^{c}\left(\phi ; \phi^{*}, \lambda_{1}\right)+g^{c}\left(\phi ;-\phi^{*}, \lambda_{1}\right)\right)+b_{2} g^{c}\left(\phi ; 0, \phi^{*}\right)
$$

where $\phi^{*}$ is the peak location calculated as in [Marschner et al. 2003]: $\phi^{*}=\phi\left(h^{*}\right)=\phi\left(\sqrt{\left(4-\eta^{\prime 2}\right) / 3}\right) ; \lambda_{1}=w_{c}$ is the width of peak, which is a user adjustable parameter to control the surface roughness. The coefficient $b_{2}$ is set as the residue value at $\phi=0$ :

$$
b_{2}=N_{T R T}(0)\left(1-g^{c}\left(0 ; \phi^{*}, w_{c}\right)\right)^{2}
$$

We observe that $b_{2}$ quickly decreases to zero as $\eta^{\prime}$ increases towards 2. For the coefficient $b_{1}$, [Marschner et al. 2003] proposed to estimate $b_{1}$ using the local curvature $d^{2} \phi / d h^{2}$. Here we propose a different estimation by preserving the energy of $N_{T R T}$, which is more physically accurate. Specifically, we estimate $b_{1}$ as:

$\frac{\int_{-\pi}^{\pi} N_{T R T}(\phi) d \phi-\int_{-\pi}^{\pi} b_{2} g^{c}(\phi) d \phi}{2 *\left(\int_{-\pi}^{\pi} g^{c}\left(\phi ; \phi^{*}, w_{c}\right) d \phi\right)} \approx \frac{\int_{-\pi}^{\pi} N_{T R T}(\phi) d \phi-b_{2} \sqrt{\pi} \phi^{*}}{2 \sqrt{\pi} w_{c}}$

where the $\int_{-\pi}^{\pi} N_{T R T}$ term is evaluated at run-time using a similar technique as described in Section 4.2.3. To do so, we first use a 4-th order Taylor expansion of the $T^{2}\left(\sigma_{a}^{\prime}\left(\theta_{d}\right), h\right)$ term (Eq 10) to factor out its dependency on $h$ :

$$
T^{2}\left(\sigma_{a}^{\prime}\left(\theta_{d}\right), h\right) \approx \sum_{k \in\{0,2,4\}} c_{k}\left(\theta_{d}, \sigma_{a}\right) h^{k}
$$

where each $c_{k}\left(\theta_{d}, \sigma_{a}\right)$ is a Taylor expansion coefficient that has an analytic expression. Next, the integration of $N_{T R T}$ over variable $\phi$ from $-\pi$ to $\pi$ can be changed to integrating over variable $h$ from -1 to 1 , which allows us to rewrite $\int_{-\pi}^{\pi} N_{T R T}$ as:

$$
\int_{-\pi}^{\pi} N_{T R T}(\phi) d \phi \approx \frac{1}{2} \sum_{k \in\{0,2,4\}} c_{k}\left(\theta_{d}, \sigma_{a}\right) \mathscr{H}_{k}^{T R T}\left(\eta, \theta_{d}\right)
$$

where $\mathscr{H}_{k}^{T R T}\left(\eta, \theta_{d}\right)=\int_{-1}^{1}\left(1-F\left(\eta, \theta_{d}, h\right)\right)^{2} F\left(\eta, \theta_{d}, h\right) h^{k} d h(k=$ $0,2,4)$ is precomputed into a $2 \mathrm{D}$ table and accessed on the fly.

When $\eta^{\prime}>2$. $N_{T R T}$ is approximated using a single Gaussian: $N_{T R T} \approx b_{3} g^{c}\left(\phi ; 0, \lambda_{3}\right)$, where the coefficient $b_{3}$ is directly set as the value of $N_{T R T}$ at $\phi=0$, and $\lambda_{3}$ is determined by preserving the energy similar to the $\eta^{\prime}<2$ case. However, this may lead to discontinuity in the representation when $\eta^{\prime}$ changes across 2 . For example, when $\eta^{\prime}<2$, the Gaussian bandwidth is $w_{c}$; and when $\eta^{\prime}>2$, the bandwidth is $\lambda_{3}$. To solve this issue, we follow [Marschner et al. 2003] to use a modified bandwidth that smoothly interpolates between $w_{c}$ and $\lambda_{3}$ over a small range $\Delta \eta^{\prime}$, whose value is typically between 0.2 and 0.4 . Specifically, the interpolated bandwidth $\lambda_{3}^{\prime}=\operatorname{interp}\left(w_{c}, \lambda_{3}, \operatorname{smoothstep}\left(2,2+\eta^{\prime}, \eta^{\prime}\right)\right)$. We also modify $b_{3}$ to $b_{3} \lambda_{3} / \lambda_{3}^{\prime}$ to preserve the energy of the lobe. 
Summary. When $\eta^{\prime}<2$, we approximate $N_{T R T}$ using 3 circular Gaussians; when $\eta^{\prime}>2$, we approximate it using one circular Gaussian. The coefficients and centers of the Gaussians are calculated through either analytic functions or query into precomputed 2D tables $\left(\mathscr{H}_{k}^{T R T}\right)$. Once $N_{T R T}$ is represented as circular Gaussian(s), we can proceed to compute $\mathscr{N}_{T R T}$ (Eq 12) as before, using the fact that the product of two circular Gaussians is still a circular Gaussian. Finally, the outer integral $\mathscr{M}_{T R T}$ is evaluated using the linear quadrature described in Section 4.2.2.

\subsubsection{Handling Eccentricity in the TRT Mode}

Normally the refractive index $\eta$ of the hair fiber is set to 1.55 . To achieve eccentricity effects due to the elliptical cross sections of real hairs, [Marschner et al. 2003] proposed to use a varying refraction index $\eta^{*}$ that depends on the azimuthal half angle $\phi_{h}$ :

$$
\begin{gathered}
\eta_{1}^{*}=2(\eta-1) a^{2}-\eta+2, \quad \eta_{2}^{*}=2(\eta-1) a^{-2}-\eta+2 \\
\eta^{*}\left(\phi_{h}\right)=\left(\left(\eta_{1}^{*}+\eta_{2}^{*}\right)+\cos \left(2 \phi_{h}\right)\left(\eta_{1}^{*}-\eta_{2}^{*}\right)\right) / 2
\end{gathered}
$$

where $a$ is the eccentricity parameter typically ranging from $0.8-$ 1.25. To incorporate eccentricity, we update and approximate the integral $\mathscr{N}_{T R T}$ (Eq 12 for the TRT mode) as:

$$
\begin{aligned}
\mathscr{N}_{T R T} & =\int g^{c}\left(\phi_{i}^{\prime} ; 0, \lambda_{j}^{\prime}\right) N_{T R T}\left(\eta^{*}\left(\phi_{h}\right), \theta_{d}, \phi_{o}-\phi_{j}-\phi_{i}^{\prime}\right) d \phi_{i}^{\prime} \\
& \approx \int g^{c}\left(\phi_{i}^{\prime} ; 0, \lambda_{j}^{\prime}\right) N_{T R T}\left(\overline{\eta^{*}}, \theta_{d}, \phi_{o}-\phi_{j}-\phi_{i}^{\prime}\right) d \phi_{i}^{\prime}
\end{aligned}
$$

where in the last step, we have substituted $\eta^{*}\left(\phi_{h}\right)$ by its average value $\overline{\eta^{*}}$, computed as:

$$
\overline{\eta^{*}}=\left(\int g^{c}\left(\phi_{i}^{\prime} ; 0, \lambda_{j}^{\prime}\right) \eta^{*}\left(\phi_{h}\right) d \phi_{i}^{\prime}\right) /\left(\int g^{c}\left(\phi_{i}^{\prime} ; 0, \lambda_{j}^{\prime}\right) d \phi_{i}^{\prime}\right)
$$

which is efficiently computed on the fly by re-using the precomputed cosine-Gaussian integral tables $\mathscr{C}_{k}$ prepared for the R mode (see Eq 14). Specifically, using the cosine's double-angle formula, we have $\cos \left(2 \phi_{h}\right)=2 \cos ^{2}\left(\phi_{h}\right)-1=2 \cos ^{2}\left(\frac{\phi_{i}^{\prime}+\phi_{j}+\phi_{o}}{2}\right)-1$. Thus we only need to access $\mathscr{C}_{0}$ and $\mathscr{C}_{2}$ to compute $\overline{\eta^{*}}$.

The purpose of the $\overline{\eta^{*}}$ substitution is to remove the dependency of $\eta^{*}\left(\phi_{h}\right)$ on the integral variable $\phi_{i}^{\prime}$, which makes it hard to model $N_{T R T}$ as a Gaussian representation. By using the average value $\overline{\eta^{*}}$, we are able to reproduce convincing eccentricity effects while incurring little modification to our existing algorithm. Fig 4 provides a comparison of our method to the ground truth.

\subsection{Computing Multiple Scattering}

In this subsection we discuss how to compute multiple scattering. Physically, multiple scattering is more complex than single scattering; however, using the dual scattering approximation [Zinke et al. 2008], multiple scattering is formulated as the sum of a global component and a local component, both of which can be incorporated into our algorithm similarly to single scattering. The key idea is again to exploit the 1D circular Gaussian representations we derived in Section 4.2 for the three scattering modes.

\subsubsection{Global Multiple Scattering}

According to Eq 3, 4 and 5, the global multiple scattering $\mathscr{M}_{t}^{G}$ for each SRBF light $j$ and each scattering mode $t$ is computed as:

$$
\begin{gathered}
\mathscr{M}_{t}^{G}=\int \psi_{f}(\cdot) S_{t}\left(\omega_{i}, \omega_{o}\right) \cos \theta_{i} d \omega_{i} \\
=\frac{1}{\cos \theta_{j}} \int \tilde{s}_{f}\left(\phi_{j}, \phi_{i}\right) g^{u}\left(\theta_{i} ; \theta_{j}, \sigma_{f}\right) M_{t}\left(\theta_{h}\right) N_{t}\left(\theta_{d}, \phi\right) \cos \theta_{i} d \omega_{i} \\
=\frac{1}{\cos \theta_{j}} \int g^{u}\left(\theta_{i} ; \theta_{j}, \sigma_{f}\right) M_{t}\left(\theta_{h}\right) \frac{\cos ^{2} \theta_{i}}{\cos ^{2} \theta_{d}} \mathscr{N}_{t}^{G}\left(\phi_{o}-\phi_{j}, \theta_{d}\right) d \theta_{i}(27)
\end{gathered}
$$

where the last step expands the integral over $d \omega_{i}$ to double integrals similar to Section 4.2; and the inner integral is denoted as $\mathscr{N}_{t}^{G}$ :

$$
\begin{aligned}
\mathscr{N}_{t}^{G}\left(\phi_{o}-\phi_{j}, \theta_{d}\right) & =\int N_{t}\left(\theta_{d}, \phi\right) \tilde{s}_{f}\left(\phi_{j}, \phi_{i}\right) d \phi_{i} \\
& =\frac{1}{\pi} \int_{\phi_{j}-\pi / 2}^{\phi_{j}+\pi / 2} N_{t}\left(\theta_{d}, \phi_{o}-\phi_{i}\right) d \phi_{i}
\end{aligned}
$$

In other words, $\mathscr{N}_{t}^{G}$ is just an integral of $N_{t}$ from $\left(\phi_{j}-\pi / 2\right)$ to $\left(\phi_{j}+\pi / 2\right)$. This is similar to the single scattering $\mathscr{N}_{t}$ defined in Eq 12, but is simpler as the $g^{c}$ term does not appear in the integral.

Thus to compute $\mathscr{N}_{t}^{G}$ for each of the R, TT, TRT modes, we can directly use our derivations of $N_{t}$ from Section 4.2. Specifically: 1) $N_{R}$ can be expressed as a sum of polynomials of $\left.\cos \phi(\mathrm{Eq} 13) ; 2\right)$ $N_{T T}$ can be approximated by a single circular Gaussian centered at $\phi=\pi(\mathrm{Eq} 16) ; 3) N_{T R T}$ can be approximated by either one circular Gaussian (when $\eta^{\prime}>2$ ) or the sum of three circular Gaussians (Eq 20, when $\eta^{\prime}<2$ ). Using these approximations, all of $N_{R}, N_{T T}$ and $N_{T R T}$ have closed form integration formulas, so $\mathscr{N}_{t}^{G}$ can be evaluated on the fly.

To evaluate the outer integral $\mathscr{M}_{t}^{G}$, we observe that the product of $g^{u}\left(\theta_{i} ; \theta_{j}, \sigma_{f}\right)$ and $M_{t}$ is still a Gaussian. Therefore we can apply the same method described in Section 4.2.2 to evaluate the quadrature of $\cos ^{2} \theta_{i} / \cos ^{2} \theta_{d} \mathscr{N}_{t}^{G}$. As this function is quite smooth, we found the approximation works very well in practice.

\subsubsection{Local Multiple Scattering}

The local multiple scattering $\mathscr{M}_{t}^{L}$ is computed as:

$$
\mathscr{M}_{t}^{L}=\frac{1}{\cos \theta_{j}} \int \tilde{s}_{f}\left(\phi_{j}, \phi_{i}\right) g^{u}\left(\theta_{i} ; \theta_{j}, \sigma_{f}\right) S_{\text {back }}\left(\omega_{i}, \omega_{o}\right) \cos \theta_{i} d \omega_{i}
$$

Here the backward scattering function $S_{\text {back }}$ is [Zinke et al. 2008]:

$S_{\text {back }}\left(\omega_{i}, \omega_{o}\right)=\frac{2}{\cos ^{2} \theta_{d}} \bar{A}_{b}\left(\theta_{d}\right) s_{b}\left(\phi_{i}, \phi_{o}\right) g^{u}\left(\theta_{i}+\theta_{o} ; \bar{\Delta}_{b}\left(\theta_{d}\right), \bar{\sigma}_{b}\left(\theta_{d}\right)\right)$

where $s_{b}\left(\phi_{i}, \phi_{o}\right)$ is $1 / \pi$ for backward scattering directions (i.e. $\cos \left(\phi_{i}-\phi_{o}\right)>0$ ), and 0 for forward scattering directions (i.e. $\left.\cos \left(\phi_{i}-\phi_{o}\right)<0\right) . \bar{A}_{b}\left(\theta_{d}\right), \bar{\Delta}_{b}\left(\theta_{d}\right), \bar{\sigma}_{b}\left(\theta_{d}\right)$ are the average backward attenuation, longitudinal shift and variance respectively. They are all 1D functions of the incident angle $\theta_{d}$.

Combining the above two equations, we can rewrite $\mathscr{M}_{t}^{L}$ as:

$$
\begin{aligned}
& \mathscr{M}_{t}^{L}=\frac{2}{\cos \theta_{j}}\left(\int \tilde{s}_{f}\left(\phi_{j}, \phi_{i}\right) s_{b}\left(\phi_{i}, \phi_{o}\right) d \phi_{i}\right) . \\
& \left(\int g^{u}\left(\theta_{i} ; \theta_{j}, \sigma_{f}\right) g^{u}\left(\theta_{i} ; \bar{\Delta}_{b}\left(\theta_{d}\right)-\theta_{o}, \bar{\sigma}_{b}\left(\theta_{d}\right)\right) \bar{A}_{b}\left(\theta_{d}\right) \frac{\cos ^{2} \theta_{i}}{\cos ^{2} \theta_{d}} d \theta_{i}\right)
\end{aligned}
$$

In other words, it is the product of two 1D integrals. The first integral, which is over $d \phi_{i}$, can be easily derived analytically:

$$
\int \tilde{s}_{f}\left(\phi_{j}, \phi_{i}\right) s_{b}\left(\phi_{i}, \phi_{o}\right) d \phi_{i}=\left(\pi-\left|\phi_{j}-\phi_{i}\right|\right) / \pi^{2}
$$

The second integral, which is over $d \theta_{i}$, is more complex. We evaluate it as follows. First, the function $g^{u}\left(\theta_{i} ; \bar{\Delta}_{b}\left(\theta_{d}\right)-\theta_{o}, \bar{\sigma}_{b}\left(\theta_{d}\right)\right)$ 
is strictly not a Gaussian, because its parameters (center and bandwidth) depend on $\theta_{d}$, which in turn depends on the variable $\theta_{i}$. To remove this dependency, our solution is to substitute $\bar{\Delta}_{b}\left(\theta_{d}\right)$ and $\bar{\sigma}_{b}\left(\theta_{d}\right)$ by their mean values computed over $\theta_{i} \in\left[\theta_{j}-\sigma_{f}, \theta_{j}+\sigma_{f}\right]$ :

$$
\widetilde{\Delta}_{b}=\frac{\int_{\theta_{j}-\sigma_{f}}^{\theta_{j}+\sigma_{f}} \bar{\Delta}_{b}\left(\frac{\theta_{i}-\theta_{o}}{2}\right) d \theta_{i}}{2 \sigma_{f}}, \widetilde{\sigma}_{b}=\frac{\int_{\theta_{j}-\sigma_{f}}^{\theta_{j}+\sigma_{f}} \bar{\sigma}_{b}\left(\frac{\theta_{i}-\theta_{o}}{2}\right) d \theta_{i}}{2 \sigma_{f}}
$$

Note that the integration range is essentially the support of the $g^{u}\left(\theta_{i} ; \theta_{j}, \sigma_{f}\right)$ function in Eq 29 . These integrals can be efficiently computed by using $1 \mathrm{D}$ SATs of $\bar{\Delta}_{b}\left(\theta_{d}\right)$ and $\bar{\sigma}_{b}\left(\theta_{d}\right)$ built on the fly.

The two mean values above allow us to define a real Gaussian function $g^{u}\left(\theta_{i} ; \widetilde{\Delta}_{b}\left(\theta_{d}\right)-\theta_{o}, \widetilde{\sigma}_{b}\left(\theta_{d}\right)\right)$, which will be further multiplied with $g^{u}\left(\theta_{i} ; \theta_{j}, \sigma_{f}\right)$ in Eq 29. This results in a new Gaussian, whose product integral with the remaining factor $\bar{A}_{b}\left(\theta_{d}\right) \cos ^{2} \theta_{i} / \cos ^{2} \theta_{d}$ can be quickly evaluated using the linear quadrature method described in Section 4.2.2.

Next, we describe how to evaluate the terms $\bar{A}_{b}\left(\theta_{d}\right), \bar{\Delta}_{b}\left(\theta_{d}\right)$, and $\left.\bar{\sigma}_{b}\left(\theta_{d}\right)\right)$ in Eq 29. According to [Zinke et al. 2008], these terms are all defined as functions of $\bar{a}_{f}\left(\theta_{d}\right), \bar{a}_{b}\left(\theta_{d}\right), \bar{\alpha}_{f}\left(\theta_{d}\right), \bar{\alpha}_{b}\left(\theta_{d}\right)$, $\bar{\beta}_{f}\left(\theta_{d}\right), \bar{\beta}_{b}\left(\theta_{d}\right)$, where $\bar{a}, \bar{\alpha}, \bar{\beta}$ stand for the average scattering attenuation, shift, and variance; and the subscripts $f / b$ indicate the forward/backward scattering respectively. These definitions can be found in Section 6 of the supplemental document. Using forward scattering as an example: the average forward attenuation $\bar{a}_{f}$ is computed by integrating the hair's scattering function $S$ over $\phi_{d}$ as well as $\omega_{o}$ :

$$
\bar{a}_{f}\left(\theta_{d}\right)=\frac{1}{\pi} \int_{\Omega_{f}} \int_{-\pi / 2}^{\pi / 2} S\left(\theta_{d}, \phi_{d}, \omega_{o}\right) \cos \theta_{d} d \phi_{d} d \omega_{o}
$$

Note that $S$ is the sum of three scattering modes. For each mode $t$, we expand the above integral by converting the outer integral (over $\Omega_{f}$ ) to double integrals over $\phi_{o}$ and $\theta_{o}$ :

$$
\begin{aligned}
\bar{a}_{f, t}= & \frac{1}{\pi} \int_{-\frac{\pi}{2}}^{\frac{\pi}{2}} \int_{\frac{\pi}{2}}^{\frac{3 \pi}{2}} \int_{-\frac{\pi}{2}}^{\frac{\pi}{2}} M_{t}\left(\left(\theta_{d}+\theta_{o}\right) / 2\right) N_{t}\left(\left(\theta_{d}-\theta_{o}\right) / 2, \phi_{o}-\phi_{d}\right) . \\
& \left(\cos \theta_{o} \cos \theta_{d}\right) / \cos ^{2}\left(\left(\theta_{d}-\theta_{o}\right) / 2\right) d \phi_{d} d \phi_{o} d \theta_{o}
\end{aligned}
$$

Noting that scattering occurs at $\theta_{o}=-\theta_{i}$, we can substitute $\left(\theta_{d}-\right.$ $\left.\theta_{o}\right) / 2$ by $\theta_{d}$, allowing us to separate the triple integral to:

$$
\begin{aligned}
\bar{a}_{f, t} \approx & \frac{1}{\pi}\left(\int_{-\frac{\pi}{2}}^{\frac{\pi}{2}} M_{t}\left(\left(\theta_{d}+\theta_{o}\right) / 2\right) \frac{\cos \theta_{o} \cos \theta_{d}}{\cos ^{2}\left(\left(\theta_{d}-\theta_{o}\right) / 2\right)} d \theta_{o}\right) . \\
& \left(\int_{\frac{\pi}{2}}^{\frac{3 \pi}{2}} \int_{-\frac{\pi}{2}}^{\frac{\pi}{2}} N_{t}\left(\theta_{d}, \phi_{o}-\phi_{d}\right) d \phi_{d} d \phi_{o}\right)
\end{aligned}
$$

In the above, the 1D integral involving $M_{t}$ can be evaluated using our linear quadrature (Section 4.2.2). The 2D integral involving $N_{t}$ is equal to $2 \int_{0}^{\pi} \phi N_{t}\left(\theta_{d}, \phi\right) d \phi$, the proof of which can be found in Section 7 of the supplemental document. Note that this integral can be analytically computed, again because we have previously modeled $N_{t}$ either as a sum of polynomials of $\cos \phi(\mathrm{R}$ mode) or circular Gaussians (TT and TRT modes), whose product integrals with $\phi$ all have analytic solutions.

Finally, the average forward scattering shift $\bar{\alpha}_{f}$ is calculated as a weighted sum of the longitudinal shifts $\alpha_{R}, \alpha_{T T}$, and $\alpha_{T R T}$ using their corresponding attenuation $\bar{a}_{f, t}$ as weights; and the average forward scattering variance $\bar{\beta}_{f}^{2}$ is the weighted sum of the longitudinal widths $\beta_{R}, \beta_{T T}$, and $\beta_{T R T}$ using the same weights. The backward scattering terms (e.g. $\bar{a}_{b, t}$ ) are similarly computed as in Eq 33, except for integrating $\omega_{o}$ over the backward scattering directions.

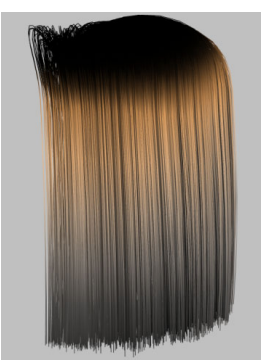

(a)

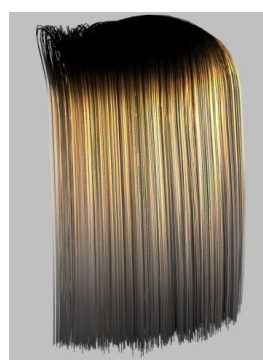

(b)

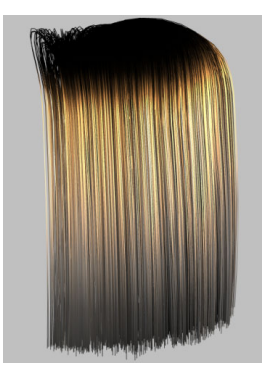

(c)
Figure 4: Result of eccentricity approximation. (a) shows circular hair (eccentricity $a=1)$; (b) shows our approximation with eccentricity $a=0.9 ;(c)$ show a reference with the same eccentricity.

\section{Implementation}

Preprocessing. In preprocessing, we follow [Ren et al. 2010] to fit SRBF lights for each environment map. Next, we need to precompute several 2D tables, including $\mathscr{C}_{k}\left(\lambda_{j}^{\prime}, \phi_{o}-\phi_{j}\right)(0 \leq k \leq 6)$ for the R mode (Eq 14); $\mathscr{H}_{k}^{T T}\left(\eta, \theta_{d}\right)(\mathrm{k}=0,2,4)$ for the TT mode (Eq 19); and $\mathscr{H}_{k}^{T R T}\left(\eta, \theta_{d}\right)(\mathrm{k}=0,2,4)$ for the TRT mode (Eq 23). For these tables, we use a resolution of 128 for the parameters $\lambda_{j}^{\prime}$ and $\eta$, which are sampled logarithmicly from the range $\lambda_{j}^{\prime} \in[0.001,10]$ and $\eta \in[1,10]$ respectively. For $\left(\phi_{o}-\phi_{j}\right)$ and $\theta_{d}$ we use a resolution of 64 . The 3 tables of $\mathscr{H}_{k}^{T T}\left(\eta, \theta_{d}\right)(\mathrm{k}=0,2,4)$ are stored as a single $2 \mathrm{D}$ texture utilizing the three color channels, so that only one texture lookup is needed to obtain all 3 values. The $\mathscr{H}_{k}^{T R T}\left(\eta, \theta_{d}\right)$ tables are stored similarly. The 7 tables of $\mathscr{C}_{k}\left(\lambda_{j}^{\prime}, \phi_{o}-\phi_{j}\right)(0 \leq$ $k \leq 6)$ are also stored as a single $2 \mathrm{D}$ texture, by placing the values corresponding to $\mathrm{k}=1,3,5$ and $\mathrm{k}=0,2,4,6$ in adjacent texels. By utilizing hardware texture interpolation, evaluating $\mathscr{N}_{R}$ only requires one texture lookup. Note that all these tables are computed only once globally, and do not need to be updated when the user changes hair scattering parameters on the fly.

Runtime Rendering. Our rendering algorithm is implemented using OpenGL shaders with multi-pass rendering. Each pass renders the result of a subset of $16 \mathrm{SRBF}$ lights, and accumulates contributions from other passes through blending. Hair fibers are rendered as line primitives. For single scattering, the specific steps are:

1. The effective transmittance $\widetilde{T}\left(\omega_{j}, \lambda_{j}\right)$ (Eq 2$)$ is obtained using an SAT of the convolution optimal depth map [Ren et al. 2010];

2. For the $\mathrm{R}$ mode, the inner integral $\mathscr{N}_{R}$ is evaluated by texture lookups into $\mathscr{C}_{k}\left(\lambda_{j}^{\prime}, \phi_{o}-\phi_{j}\right)(\mathrm{Eq} 14)$;

3. For the TT and TRT modes, the fitted circular Gaussian parameters are first estimated from the two energy terms $\int_{-\pi}^{\pi} N_{T T}$ and $\int_{-\pi}^{\pi} N_{T R T}$, which are evaluated via texture lookups into $\mathscr{H}_{k}^{T T}\left(\eta, \theta_{d}\right)$ and $\mathscr{H}_{k}^{T R T}\left(\eta, \theta_{d}\right)$ (Eq 19 and 23). Following this, each inner integral $\mathscr{N}_{T T}$ and $\mathscr{N}_{T R T}$ is computed as the product of two circular Gaussians using the analytic formula. To account for eccentricity in the TRT mode, a modified index of refraction $\overline{\eta^{*}}$ is calculated via texture lookups into $\mathscr{C}_{k}\left(\lambda_{j}^{\prime}, \phi_{o}-\phi_{j}\right)(\mathrm{Eq} 26)$, and is used when evaluating $\mathscr{N}_{T R T}$;

4. Finally, we employ the linear quadrature method (Section 4.2.2) to evaluate the outer integrals $\mathscr{M}_{t}$ (Eq 11). Depending on the number of samples $m+1$, this requires multiple evaluations of $\mathscr{N}_{R}, \mathscr{N}_{T T}$, and $\mathscr{N}_{T R T}$, which are obtained in steps $2-3$.

To compute multiple scattering, we first obtain the forward scattering transmittance $T_{f}\left(\omega_{j}\right)$ and spread $\bar{\sigma}_{f}^{2}\left(\omega_{j}\right)$ (Eq 3 and 5) using a sparse sampling approach proposed in [Ren et al. 2010]. We then compute the global and local multiple scattering components 


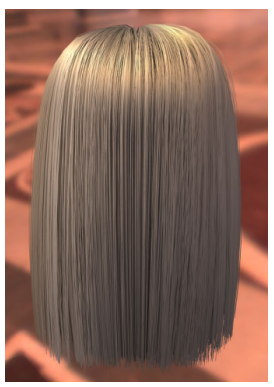

(a) reference

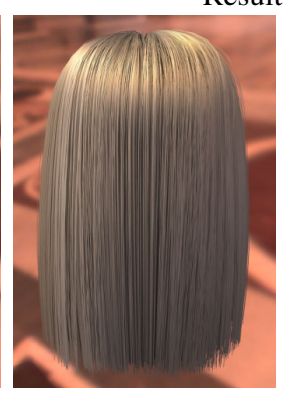

(b) $m=6$
Result images

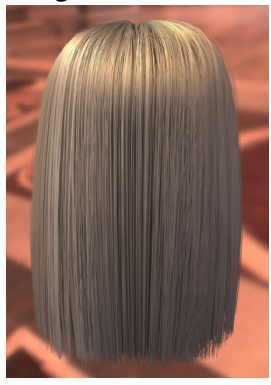

(c) $m=4$

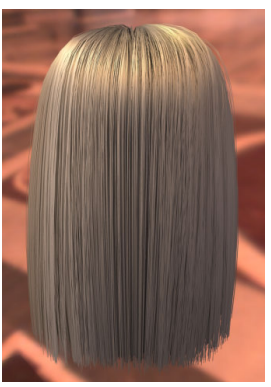

(d) $m=2$

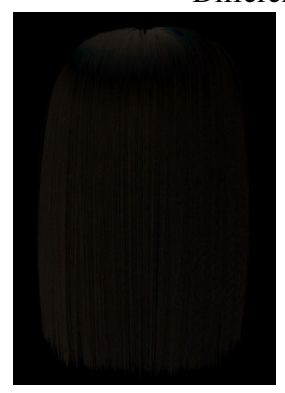

(e) $m=4$
Differences $\times 8$

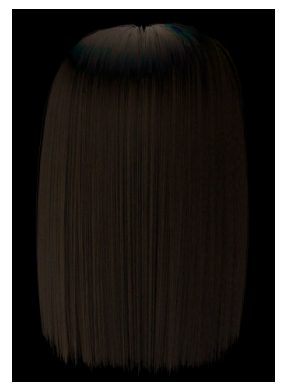

(f) $m=2$

Figure 5: Results of our linear quadrature method described in Section 4.2.2. (a) is computed using our method with a large number of segments $m=200$, which we treat as a reference; $(b, c, d)$ show the results using a much smaller number of segments: $m=6,4,2$ respectively; $(e)$ and $(f)$ show the magnified difference images of the $m=4$ and 2 cases with the reference. Note that at $m=2$, our result has subtle but observable differences compared to the reference; and at $m=4$, our result is almost indistinguishable from the reference.

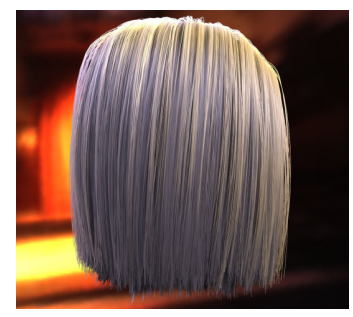

(a) our S.S

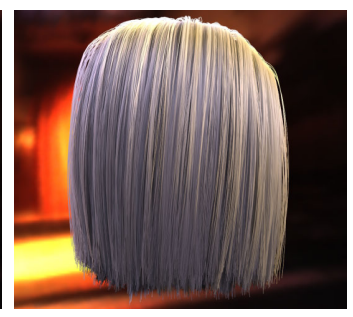

(b) S.S. by Ren et al.

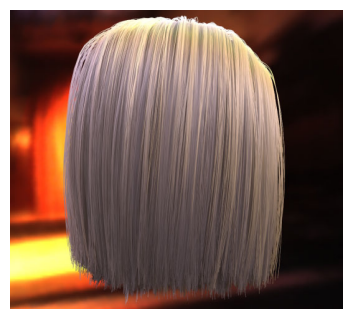

(c) our S.S.+M.S.

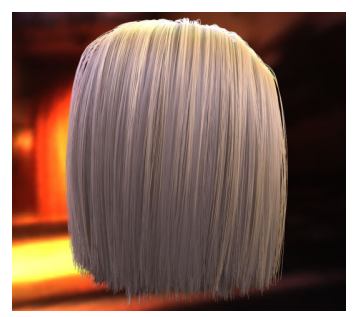

(d) S.S.+M.S. by Ren et al.

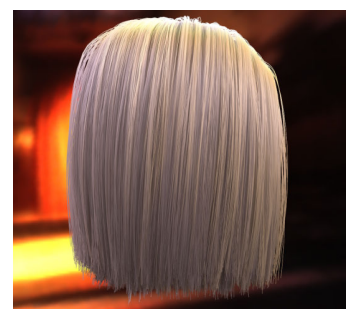

(e) reference

Figure 6: Comparison of our method to [Ren et al. 2010] and reference. (a) and (b) compare the single scattering (S.S.) effect; (c) and (d) compare the full single + multiple scattering (S.S. + M.S.) effect; $(e)$ is a reference image including both single and multiple scattering effects generated using a photon mapping approach [Moon and Marschner 2006]. In both our method and [Ren et al. 2010], the environment light is approximated by 41 SRBF lights. Note that the images match well visually and the differences are subtle.

separately. Computing the global multiple scattering integral $\mathscr{M}_{t}^{G}$ (Eq 27) is very similar to computing the single scattering integral, thus they share largely the same steps as described above.

To compute local multiple scattering $\mathscr{M}_{t}^{L}$ (Eq 29), we first sample the average terms $\bar{A}_{b}\left(\theta_{d}\right), \bar{\Delta}_{b}\left(\theta_{d}\right), \bar{\sigma}_{b}\left(\theta_{d}\right)$ into a 1D texture of resolution 128, and compute the SATs of $\bar{\Delta}_{b}\left(\theta_{d}\right)$ and $\bar{\sigma}_{b}\left(\theta_{d}\right)$. These then allow us to obtain the mean values $\widetilde{\Delta}_{b}$ and $\widetilde{\sigma}_{b}$ via texture lookups. Finally we use the linear quadrature again to evaluate $\mathscr{M}_{t}^{L}$. Note that the $1 \mathrm{D}$ textures are computed and updated on the fly whenever the hair scattering parameters are modified. The total computation overhead is very small.

In sum, as we choose $m=4$ piecewise linear segments, for each SRBF light, computing single scattering at any shading point requires a total of 14 texture lookups, and multiple scattering requires 11 texture lookups. Currently, we do not incorporate the view transparency effect, which can be easily added using techniques such as occupancy maps [Sintorn and Assarsson 2009].

\section{Comparisons and Results}

In this section we present results of our method and comparisons to previous work. Many hair geometry models are courtesy of Cem Yuksel et al. [2008], Zinke et al. [2008], and Selle et al. [2008]. Our parameters and units are set by following [Marschner et al. 2003].

Accuracy. To verify the accuracy of our method, we first mathematically analyze the proposed 1D circular Gaussian representation. In Fig $3(\mathrm{a}, \mathrm{b})$ we compare our approximated $N_{T T}$ and $N_{T R T}$ functions to the ground truth computed at three different incident angles $\theta_{d}=0, \pi / 6$ and $\pi / 3$. In (c) we compare the 4-th order Taylor expansion of the attenuation factor $T$ to the ground truth com-

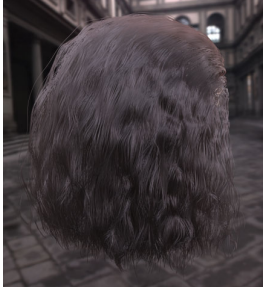

(a) $(-0.05,0.16)$

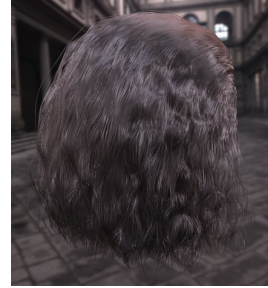

(b) $(-0.05,0.09)$

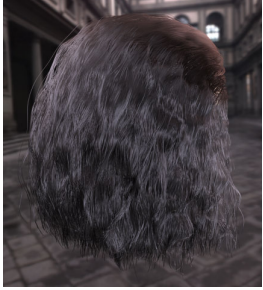

(c) $(-0.34,0.09)$
Figure 7: Editing the longitudinal shift and width $\left(\alpha_{R}, \beta_{R}\right)$. Note the resulting change in the hair's highlights.

puted with three difference absorption coefficients $\sigma_{a}=0.2,0.6$ and 1.0. Note that in all examples, our approximations match the references very well. We do not plot the $N_{R}$ function because it does not involve a circular Gaussian approximation.

Next, we examine the linear quadrature method described in Section 4.2.2. Recall that we use it to compute all product integrals of 1D Gaussian with a function in the form of $\mathscr{N}_{t}\left(\lambda_{j}^{\prime}, \phi_{o}-\right.$ $\left.\phi_{j}, \theta_{d}\right) \cos ^{2} \theta_{i} / \cos ^{2} \theta_{d}$, where $t \in(R, T T, T R T)$. Examples of these functions, at parameter settings $\lambda_{j}^{\prime}=0.5$ and $\phi_{o}-\phi_{j}=0$ or $\pi$, are plotted in Fig 3(d). The cases of $N_{T T}$ at $\phi_{o}-\phi_{j}=0$ and $N_{T R T}$ at $\phi_{o}-\phi_{j}=\pi$ are omitted because they are almost zero. As illustrated, these functions are quite smooth, requiring only a few samples (segments) to accurately evaluate their integrals with a Gaussian. Additional plots of these functions are provided in Section 9 of the supplemental document. Fig 5 shows the rendering results computed using our method with different number of segments $m$, and a comparison to ground truth. Observe that at the default choice of $m=4$, our result is almost indistinguishable from the reference. 


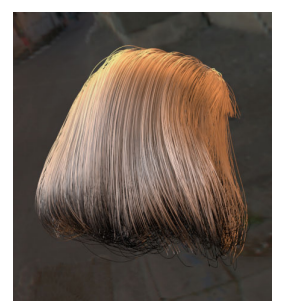

(a) $(0.03,0.37)$

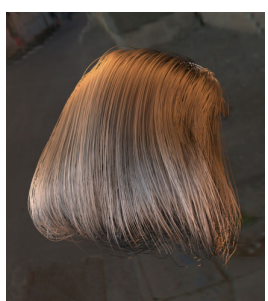

(b) $(-0.26,0.37)$

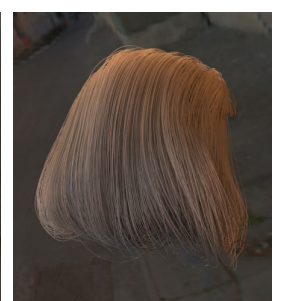

(c) $(-0.26,0.7)$

Figure 8: Editing the longitudinal shift and width $\left(\alpha_{R}, \beta_{R}\right)$.

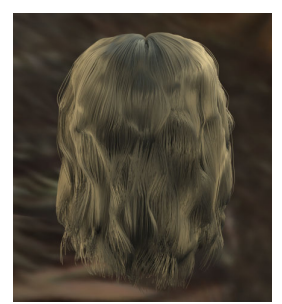

(a)

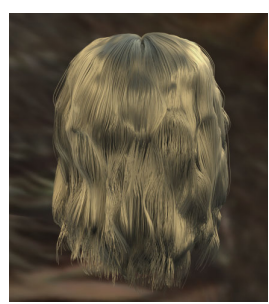

(b)

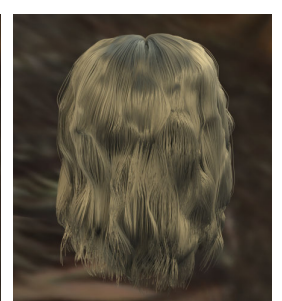

(c)

Figure 9: Editing index of refraction $\eta$ and the azimuthal width $w_{c}$ for the TRT caustic. (a) $\eta=1.54, w_{c}=0.06$; (b) $\eta=1.65$, $w_{c}=0.06 ;$; $(c) \eta=1.65, w_{c}=0.20$.

In Fig 4 we evaluate the approximated eccentricity described in Section 4.2.5. Our approximation produces visually matching result to the reference, which is computed by using the accurate eccentricity factor and summing up 1536 directional lights in a bruteforce way. By accounting for eccentricity, we allow the user to dynamically edit this parameter, resulting in realistic and interesting variations in the hair appearance [Marschner et al. 2003].

Finally, in Fig 6, we compare our rendering results to the method presented by [Ren et al. 2010] and the reference generated by photon mapping [Moon and Marschner 2006]. Note that for both single and multiple scattering, the results look qualitatively the same and the differences are subtle. However, our method allows for the dynamic editing of all scattering parameters in the Marschner model, while Ren et al. require fixing these parameters at precomputation time. Section 10 in the supplemental document provides additional comparisons between our method and [Ren et al. 2010] under a single SRBF light at different bandwidth and comparisons between renderings computed with directional lights vs. SRBF lights.

Performance. All results are obtained on a PC with Intel Core 2 Duo $3.00 \mathrm{GHz}$ CPU, 6 GB RAM and an NVIDIA GTX 580 GPU. The image resolution is $720 \times 480$. The rendering frame rates are reported in Table 2 . The rendering cost is roughly proportional to the piecewise linear segments $m$, which we set to 4 by default. In all examples we are able to achieve interactive frame rates.

Parameter editing. We show several examples of editing the hair scattering parameters on the fly. All parameters defined in the Marschner model can be modified. In Fig 1 we show that the user paints directly onto the hair model to edit the spatially-varying absorption coefficients. This results in a dynamic simulation of hair coloring computed on the fly. In Fig 7, we edit longitudinal shift $\alpha_{R}$ and width $\beta_{R}$, and the other parameters $\alpha_{T T}=-\alpha_{R} / 2$, $\alpha_{T R T}=-3 \alpha_{R} / 2, \beta_{T T}=\beta_{R} / 2, \beta_{T R T}=2 \beta_{R}$ are updated accordingly. This simulates changing the tilting angle the hair scales. In particular, in Fig 7(b), the longitudinal width $\beta_{R}$ is reduced, resulting in sharper specular highlights. In Fig 7(c), the longitudinal shift $\alpha_{R}$ is reduced, causing the specular highlights shift downward. Fig 8 gives another example of editing longitudinal shift and width.

Fig 9 shows the editing of the index of refraction and the azimuthal

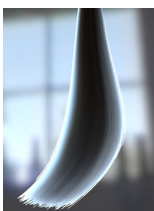

(a)

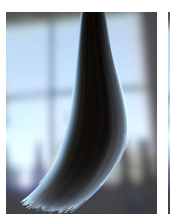

(b)

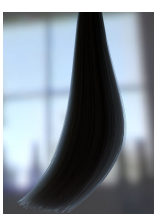

(c)

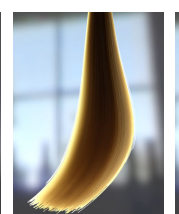

(d)

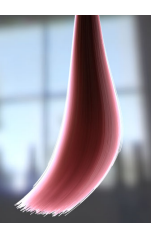

(e)
Figure 10: Editing the absorption coefficient $\sigma_{a}$. (a) $\sigma_{a}=$ $(0.36,0.36,0.36) ;(b) \sigma_{a}=(0.55,0.55,0.55) ;$ (c) $\sigma_{a}=(1,1,1) ;(d)$ $\sigma_{a}=(0.27,0.39,0.65) ;\left(\right.$ e) $\sigma_{a}=(0.23,0.42,0.42)$. Note the change in colors and back lighting effects due to the TT component.

\begin{tabular}{ccccc}
\hline data & \#fibers & \#points & \#SRBFs & FPS \\
\hline animation (Fig 1) & $10 \mathrm{k}$ & $270 \mathrm{k}$ & 40 & 8.3 \\
straight (Fig 6) & $50 \mathrm{k}$ & $1.25 \mathrm{M}$ & 41 & 5.9 \\
natural (Fig 7) & $10 \mathrm{k}$ & $1.5 \mathrm{M}$ & 40 & 5.8 \\
bob cut (Fig 8) & $10 \mathrm{k}$ & $350 \mathrm{k}$ & 30 & 10.6 \\
dark (Fig 9) & $15 \mathrm{k}$ & $1.0 \mathrm{M}$ & 36 & 6.2 \\
ponytail (Fig 10) & $6 \mathrm{k}$ & $100 \mathrm{k}$ & 42 & 8.9 \\
\hline
\end{tabular}

Table 2: Performance of examples demonstrated in our paper. The timing includes both single and multiple scattering computations.

width $w_{c}$ for the TRT caustic. Observe that 1) increasing $\eta$ in (b) results in a brighter reflection due to the larger Fresnel reflection term; and 2) increasing $w_{c}$ in (c) results in smoother specular highlights. Finally, Fig 10 shows the editing of the absorption coefficient, resulting in color changes and back lighting effects caused by the TT component. Please refer to the paper video for additional results.

\section{Conclusions}

To summarize, we have presented a new method for interactive hair rendering and appearance editing under environment lighting represented as SRBF lights. We derive a compact 1D circular Gaussian representation for the hair scattering function, allowing us to compute their closed-form integrals with SRBF lights at run-time. We provide a GPU implementation that achieves interactive rendering and editing rates for spatially-varying hair parameters and arbitrary environment maps. Such capability is important for design and prototyping applications, and has not been achieved by previous work.

There are several directions for future work. First, we would like to incorporate the artist-friendly model by [Sadeghi et al. 2010]. Since this model is fundamentally based on Marschner's model, the dual scattering approximation, and the use cosine/Gaussian functions to approximate the R, TT, TRT azimuthal functions, it can be directly combined with SRBF lighting using our proposed method. Second, we would like to extend our work to near-field light sources in a global illumination environment, and are also interested in handling furs represented by texture layers [Silva et al. 2010]. Finally, our 1D circular Gaussian representation is not limited to real-time applications - they can also benefit high-quality offline renderings, as they provide a concise and accurate substitute for the original hair scattering function.

Acknowledgments. We thank SIGGRAPH anonymous reviewers for their comments and suggestions. This work was supported by the National Basic Research Project of China (2011CB302205), the Natural Science Foundation of China (61120106007), and the National Science Foundation of the United States (CCF-0746577).

\section{References}

Ben-Artzi, A., Overbeck, R., AND RAMAmoorthi, R. 2006. Real-time BRDF editing in complex lighting. ACM Trans. Graph. 25, 3, 945-954. 
Bonneel, N., Paris, S., van de Panne, M., Durand, F., AND DRETTAKIS, G. 2009. Single photo estimation of hair appearance. Computer Graphics Forum 28, 4, 1171C-1180.

Debevec, P. E., AND Malik, J. 1997. Recovering high dynamic range radiance maps from photographs. In Proc. of ACM SIGGRAPH, 369-378.

D'Eon, E., Francois, G., Hill, M., LetTeri, J., AND Aubry, J.-M. 2011. An energy-conserving hair reflectance model. Computer Graphics Forum 30, 4, 1181-1187.

Green, P., Kautz, J., And Durand, F. 2007. Efficient reflectance and visibility approximations for environment map rendering. Computer Graphics Forum 26, 3, 495-502.

Jakob, W., Moon, J. T., AND Marschner, S. 2009. Capturing hair assemblies fiber by fiber. ACM Trans. Graph. 28, 5, 164.

KAJIYA, J. T., AND KAY, T. L. 1989. Rendering fur with three dimensional textures. In Proc. of ACM SIGGRAPH, 271-280.

Kim, T.-Y., And Neumann, U. 2001. Opacity shadow maps. In Proc. of Eurographics Rendering Workshop, 177-182.

Lokovic, T., And Veach, E. 2000. Deep shadow maps. In Proc. of ACM SIGGRAPH, 385-392.

Marschner, S. R., Jensen, H. W., Cammarano, M., WorLEY, S., AND HANRAHAN, P. 2003. Light scattering from human hair fibers. ACM Trans. Graph. 22, 3, 780-791.

Mertens, T., Kautz, J., Bekaert, P., and Van Reeth, F. 2004. A self-shadow algorithm for dynamic hair using density clustering. In SIGGRAPH 2004 Sketches, 44.

Moon, J. T., And Marschner, S. R. 2006. Simulating multiple scattering in hair using a photon mapping approach. ACM Trans. Graph. 25, 3, 1067-1074.

Moon, J. T., Walter, B., And Marschner, S. 2008. Efficient multiple scattering in hair using spherical harmonics. $A C M$ Trans. Graph. 27, 3, 31:1-31:7.

Navarro, F., Gutierrez, D., And Sern, F. 2009. Interactive hdr lighting of dynamic participating media. The Visual Computer 25, 4, 339-347.

Paris, S., Chang, W., Kozhushnyan, O. I., Jarosz, W., MATUSiK, W., ZWICKER, M., AND DURAND, F. 2008. Hair Photobooth: geometric and photometric acquisition of real hairstyles. ACM Trans. Graph. 27, 3, 30:1-30:9.

Ren, Z., Zhou, K., Li, T., HuA, W., And Guo, B. 2010. Interactive hair rendering under environment lighting. ACM Trans. Graph. 29, 4, 55:1-55:8.

Sadeghi, I., Pritchett, H., Jensen, H. W., And Tamstorf, R. 2010. An artist friendly hair shading system. ACM Trans. Graph. 29, 4, 56:1-56:10.

SCHLICK, C. 1994. An inexpensive BRDF model for physicallybased rendering. Computer Graphics Forum 13, 3, 233-246.

Selle, A., Lentine, M., And FedKiw, R. 2008. A mass spring model for hair simulation. ACM Trans. Graph. 27, 3, 64.

Shinya, M., Shiraishi, M., Dobashi, Y., Iwasaki, K., AND NishiTA, T. 2010. A simplified plane-parallel scattering model and its application to hair rendering. Pacific Conference on Computer Graphics and Applications, 85-92.

Silva, P., Bando, Y., Chen, B.-Y., And Nishita, T. 2010. Curling and clumping fur represented by texture layers. The $\mathrm{Vi}$ sual Computer 26, 6, 659-667.
Sintorn, E., AND ASSARSSON, U. 2008. Real-time approximate sorting for self shadowing and transparency in hair rendering. In Proc. of I3D, 157-162.

Sintorn, E., AND Assarsson, U. 2009. Hair self shadowing and transparency depth ordering using occupancy maps. In Proc. of $I 3 D, 67-74$.

Sun, X., Zhou, K., Chen, Y., Lin, S., Shi, J., And Guo, B. 2007. Interactive relighting with dynamic BRDFs. ACM Trans. Graph. 26, 3 .

Sun, X., Zhou, K., Stollnitz, E., Shi, J., And Guo, B. 2008. Interactive relighting of dynamic refractive objects. ACM Trans. Graph. 27, 3, 1-9.

TSAI, Y.-T., AND SHIH, Z.-C. 2006. All-frequency precomputed radiance transfer using spherical radial basis functions and clustered tensor approximation. ACM Trans. Graph. 25, 3, 967-976.

Wang, R., Cheslack-Postava, E., Wang, R., Luebke, D., Chen, Q., Hua, W., Peng, Q., And BaO, H. 2008. Real-time editing and relighting of homogeneous translucent materials. Vis. Comput. 24, 7, 565-575.

Wang, J., Ren, P., Gong, M., Snyder, J., And Guo, B. 2009. All-frequency rendering of dynamic, spatially-varying reflectance. ACM Trans. Graph. 28, 5, 133:1-133:10.

WArd, K., Bertails, F., Kim, T.-Y., Marschner, S. R., CANI, M.-P., AND LIN, M. C. 2007. A survey on hair modeling: Styling, simulation, and rendering. IEEE Transactions on Visualization and Computer Graphics 13, 2, 213-234.

XU, K., GaO, Y., LI, Y., JU, T., AND HU, S.-M. 2007. Real-time homogenous translucent material editing. Computer Graphics Forum 26, 3, 545-552.

Yuksel, C., AND Keyser, J. 2008. Deep opacity maps. Computer Graphics Forum 27, 2, 675-680.

ZINKE, A., AND WEBER, A. 2006. Global illumination for fiber based geometries. In SIACG 2006.

ZINKE, A., AND WeBER, A. 2007. Light scattering from filaments. IEEE Transactions on Visualization and Computer Graphics 13, 2, 342-356.

Zinke, A., Yuksel, C., Weber, A., And Keyser, J. 2008. Dual scattering approximation for fast multiple scattering in hair. ACM Trans. Graph. 27, 3, 32:1-32:10.

Zinke, A., Rump, M., Lay, T., Weber, A., Andriyenko, A., AND KLEIN, R. 2009. A practical approach for photometric acquisition of hair color. ACM Trans. Graph. 28, 5, 165:1-165:9.

\section{Appendix}

Explanation of Eq 11. (Separation of SRBF $G_{j}$ into two 1D circular Gaussian). First, let's express the directions $\omega_{i}$ and $\omega_{j}$ in Euclidean coordinates:

$$
\begin{aligned}
\omega_{i} & =\left[\sin \theta_{i}, \cos \theta_{i} \cos \phi_{i}, \cos \theta_{i} \sin \phi_{i}\right] \\
\omega_{j} & =\left[\sin \theta_{j}, \cos \theta_{j} \cos \phi_{j}, \cos \theta_{j} \sin \phi_{j}\right]
\end{aligned}
$$

Then, the dot product of $\omega_{i}$ and $\omega_{j}$ can be written as (using productto-sum rules in trigonometry):

$$
\omega_{i} \cdot \omega_{j}-1=\left[\cos \left(\theta_{i}-\theta_{j}\right)-1\right]+\cos \theta_{i} \cos \theta_{j}\left[\cos \left(\phi_{i}-\phi_{j}\right)-1\right]
$$

Now divide both sides by $\lambda_{j}^{2} / 2$, and apply the exponential function: $G\left(\omega_{i} ; \omega_{j}, \lambda_{j}\right)=g^{c}\left(\theta_{i} ; \theta_{j}, \lambda_{j}\right) \cdot g^{c}\left(\phi_{i} ; \phi_{j}, \lambda_{j} / \sqrt{\cos \left(\theta_{i}\right) \cos \left(\theta_{j}\right)}\right)$ 